\title{
Personalizing Antithrombotic Therapy in COVID-19: Role of Thromboelastography and Thromboelastometry
}

\author{
Rahul Chaudhary ${ }^{1,2}$ Rolf P. Kreutz ${ }^{3}$ Kevin P. Bliden ${ }^{2}$ Udaya S. Tantry ${ }^{2}$ Paul A. Gurbel ${ }^{2}$ \\ ${ }^{1}$ Division of Hospital Internal Medicine, Mayo Clinic, Rochester, \\ Minnesota, United States \\ 2 Sinai Center of Thrombosis Research, Sinai Hospital of Baltimore, \\ Baltimore, Maryland, United States \\ ${ }^{3}$ Department of Internal Medicine, Krannert Institute of Cardiology, Indiana \\ University School of Medicine, Indianapolis, Indiana, United States \\ Address for correspondence Rahul Chaudhary, MD, FACP, Division of \\ Hospital Internal Medicine, Department of Internal Medicine, Mayo \\ Clinic, 200 First Street SW, Rochester MN 55905, United States \\ (e-mail: Chaudhary.rahul@mayo.edu; \\ rahulchaudhary1234@gmail.com).
}

Thromb Haemost 2020;120:1594-1596.

Coronavirus-2019 (COVID-19) infection is responsible for the pandemic outbreak across the world. The pathophysiology of COVID-19 has been characterized by endothelial dysfunction, and a thromboinflammatory state. Severe infection and poor clinical outcomes have been associated with diffuse endothelial dysfunction and a hyperinflammatory state leading to a cytokine storm which further enhances thrombotic complications. ${ }^{1,2}$ The pathophysiology uniquely affects the lungs in its early stages as evidenced by autopsy reports of diffuse alveolar damage along with pulmonary intravascular microthrombi in the absence of known venous thromboembolism (VTE). ${ }^{3}$ Multiple retrospective and prospective clinical trials have evaluated thromboinflammatory biomarkers linking them to poor prognosis among patients with COVID-19 infection. ${ }^{4-6}$ The thrombotic biomarkers evaluated include D-dimer levels, fibrinogen levels, prothrombin time (PT), and activated partial thromboplastin time (aPTT). There have been several reports and meta-analyses linking elevated D-dimer levels with poor prognosis including severity and mortality with COVID-19 infection. ${ }^{5,7}$ However, several concerns have been raised with the use of D-dimer as a biomarker among COVID-19 infected patients. D-dimer has low specificity and elevated levels are often seen with advanced age, African American race, female sex, active malignancy, surgery, pregnancy, immobility, cocaine use, connective tissue disorders, end-stage renal disease, and prior thromboembolic disease. ${ }^{8}$ Also, D-dimer reflects a later stage in the hemostatic process and is released when a clot is degraded by the fibrinolytic processes. Other standard laboratory tests including PT and aPTT measure the clotting activity from the plasma and ignore other components of the coagulation such as the platelets and the fibrinolysis. The platelet count and fibrinogen concentration also have the

received

June 12, 2020

accepted

June 18,2020

caveat of providing static measures with no information regarding their functionality.

On the other hand, whole blood viscoelastic analysis can be rapidly performed by thromboelastography (TEG) or rotational thromboelastometry (ROTEM), which measure the whole blood capability to make and sustain clot formation. ${ }^{9}$ Several reports have now been published describing early experience with TEG or ROTEM for patients admitted with severe COVID-19 to critical care settings in Italy and the United States. ${ }^{10-12}$ The TEG parameters include reaction time $(R$, represents the initiation phase and measures the time of latency from start of the test to initial fibrin formation), clot formation time ( $K$, represents amplification phase and measures the time taken to achieve $20 \mathrm{~mm}$ of clot strength), angle or $\alpha$ ( $K$ angle, represents the propagation phase and measures the rate of clot formation), maximum amplitude (MA, represents the overall stability of the clot), and amplitude at 30 minutes (LY30, represents the fibrinolysis phase and measures the percentage decrease in amplitude at 30 minutes post-MA). Panigada et al demonstrated a hypercoagulable profile measured by TEG in 24 patients admitted to the intensive care unit (ICU) with COVID-19. ${ }^{10}$ They noted that the reaction time $(R)$ and clot formation time $(K)$ were shorter than those of the reference population in 50 and $83 \%$ of the COVID-19 patients, respectively. $K$ angle and MA values were higher than those of the reference population in 72 and $83 \%$ of the COVID-19 patients, whereas clot lysis at 30 minutes (LY-30) was lower in 100\% of the COVID19 population. ${ }^{10}$ Similar results were observed by Maatman et al and Wright et al, showing a COVID-19-associated hypercoagulable state measured by TEG and associated fibrinolysis shutdown (defined by LY-30 $<0.8 \%)^{11}$ in 12 and

(c) 2020 Georg Thieme Verlag KG Stuttgart · New York
DOI https://doi.org/ 10.1055/s-0040-1714217. ISSN 0340-6245. 
Table 1 Summary of TEG and ROTEM parameters in COVID-19 infection (data presented as mean values in COVID-19/reference range)

\begin{tabular}{|c|c|c|c|c|c|c|}
\hline \multicolumn{7}{|l|}{ TEG } \\
\hline & $R(\min )$ & $K(\min )$ & Angle $K$ (degree) & $\mathrm{MA}(\mathrm{mm})$ & LY30 (\%) & D-dimer $(\mathrm{ng} / \mathrm{mL})$ \\
\hline Panigada et al ${ }^{10}(n=24)$ & $6.3 / 4-8$ & $1.5 / 0-4$ & $69.4 / 47-74$ & $79.1 / 54-72$ & $7.8 / 0-8$ & $4,877 \mid<500$ \\
\hline Wright et a ${ }^{11}(n=44)$ & $5.8 / 2-8$ & $\mathrm{~N} / \mathrm{A}$ & $71 / 55-78$ & $73 / 50-70$ & $0 / 0.8-3$ & $1,840 /<500$ \\
\hline Maatman et $\mathrm{a}^{12}(n=12)$ & $4.8 / 5-11$ & $1.4 / 1-3$ & $69.6 / 53-73$ & $70.8 / 50-72$ & $0.8 / 0-7$ & $506 / 321-973$ \\
\hline Mortus et al ${ }^{13}(n=21)$ & $6 / 4-7$ & $\mathrm{~N} / \mathrm{A}$ & $73 / 61-73$ & $74 / 55-65$ & $2.1 / 0-5$ & 8,300 \\
\hline \multicolumn{7}{|l|}{ ROTEM } \\
\hline & $C T(s)$ & CFT (s) & Angle $K$ (degree) & MCF (mm) & ML (\%) & D-dimer $(\mathrm{ng} / \mathrm{mL})$ \\
\hline Pavoni et al ${ }^{14}(n=40)$ & $78.3 / 38-79$ & $41.6 / 34-159$ & $\mathrm{~N} / \mathrm{A}$ & $76.6 / 50-72$ & 9.4 & 1,556 \\
\hline
\end{tabular}

Abbreviations: CFT, clot formation time; CT, clotting time; $K$, clot formation time; LY30, amplitude at 30 minutes; MA, maximum amplitude; MCF, maximum clot firmness; ML, maximum lysis; $R$, reaction time; ROTEM, rotational thromboelastometry; TEG, thromboelastography.

44 patients admitted to the ICU, respectively (- Table 1). ${ }^{11,12}$ The patients with hypercoagulable profile and/or fibrinolysis shutdown were found to have a higher rate and shorter time to VTE ( 40 vs. $5 \%$ in patients without shutdown, $p=0.013$ ). ${ }^{12}$ Similarly, Mortus et al found high $K / \alpha$ or high MA in 19 out of 21 patients admitted with severe COVID-19 when measured by TEG. ${ }^{13}$ Finally, Pavoni et al showed consistent evidence of a hypercoagulable state in severe COVID-19 with the ROTEM platform with improvement of fibrinogen clot firmness measurements after 10 days of illness, suggesting a dynamic component of changes in coagulation that accompany the rise and fall of inflammatory parameters. ${ }^{14}$

In summary, all the current studies support COVID-19 as a hypercoagulable and hypofibrinolytic state in the ICU setting. It should be noted that all the above-mentioned studies used the TEG5000 hemostasis analyzer (Haemonetics, Braintree, Massachusetts, United States) instead of the TEG-6S, which is a point-of-care device and did not measure fibrin clot strength. ${ }^{9}$ Prior studies have shown fibrin clot strength as a strong and independent predictor of ischemic outcomes. ${ }^{15}$

All intubated ICU patients with low risk of bleeding are recommended low-intensity pharmacologic prophylaxis including low-molecular-weight heparin (nadroparin 65 IU/ $\mathrm{kg} /$ day, or dalteparin 5,000 IU/day, or enoxaparin $40 \mathrm{mg} /$ day) and low-dose unfractionated heparin (5,000 units twice a day) for VTE prevention. ${ }^{16}$ However, due to the very high incidence of thromboembolic complications despite standard low-dose VTE prophylaxis among severe COVID-19 infections with elevated D-dimer levels, intermediate-intensity or full-dose anticoagulation as opposed to standard low-intensity prophylaxis is now routinely used. ${ }^{17}$ The data regarding the best biomarkers to guide decisions about intensification of anticoagulant prophylaxis in patients with severe COVID-19 are rapidly evolving. ${ }^{17}$ Due to the ease of testing and their properties as a single test that is able to evaluate different components and stages of coagulation and platelet function at the bedside, TEG or ROTEM may be ideally suited to predict thrombotic risk in patients with moderate or severe COVID-19. Other advantage, especially in the setting of COVID-19, includes the flexibility of point-of-care testing with the newer TEG6s and ROTEM-Sigma devices, which can be safely performed without the risk of aerosolization. To this end, we are conducting an ongoing study-The evaluation of hemostasis by thromboelastography, platelet function testing, and biomarker analysis in hospitalized COVID-19 patients (TARGET-COVID Study)-to determine an individual patient's degree of thrombotic and bleeding risk using TEG. We propose that the development of individual thrombogenic phenotypes will help personalize antithrombotic therapy in patients affected by COVID-19 and improve outcomes.

Funding

None.

Conflict of Interest

R.P.K. has received consulting fees from Haemonetics. P.A. G. receives honoraria for lectures and consultations, including service on speakers' bureaus from Bayer, Merck, Janssen, Medicure, and World Medical, and is receiving grants from the National Institutes of Health, Janssen, Merck, Bayer, Haemonetics, Instrumentation Labs, and Amgen. P.A.G. holds stock or stock options in Merck, Medtronic, and Pfizer, and is holding patents in the area of personalized antiplatelet therapy and interventional cardiology. Other authors report no disclosure.

\section{References}

1 Huang C, Wang Y, Li X, et al. Clinical features of patients infected with 2019 novel coronavirus in Wuhan, China. Lancet 2020;395 (10223):497-506

2 Varga Z, Flammer AJ, Steiger P, et al. Endothelial cell infection and endotheliitis in COVID-19. Lancet 2020;395(10234):14171418

3 Fox SE, Akmatbekov A, Harbert JL, Li G, Quincy Brown J, Vander Heide RS. Pulmonary and cardiac pathology in African American patients with COVID-19: an autopsy series from New Orleans. Lancet Respir Med 2020;8(07):681-686

4 Tang N, Li D, Wang X, Sun Z. Abnormal coagulation parameters are associated with poor prognosis in patients with novel coronavirus pneumonia. J Thromb Haemost 2020;18(04):844-847

5 Tang N, Bai H, Chen X, Gong J, Li D, Sun Z. Anticoagulant treatment is associated with decreased mortality in severe coronavirus disease 2019 patients with coagulopathy. J Thromb Haemost 2020;18(05):1094-1099 
6 Qin C, Zhou L, Hu Z, et al. Dysregulation of immune response in patients with COVID-19 in Wuhan, China. Clin Infect Dis 2020. Doi: $10.1093 / \mathrm{cid} / \mathrm{ciaa} 248$

7 Lippi G, Favaloro EJ. D-dimer is associated with severity of coronavirus disease 2019: a pooled analysis. Thromb Haemost 2020;120(05):876-878

8 Kabrhel C, Mark Courtney D, Camargo CA Jr, et al. Factors associated with positive D-dimer results in patients evaluated for pulmonary embolism. Acad Emerg Med 2010;17(06):589-597

9 Gurbel PA, Bliden KP, Tantry US, et al. First report of the point-ofcare TEG: a technical validation study of the TEG-6S system. Platelets 2016;27(07):642-649

10 Panigada M, Bottino N, Tagliabue P, et al. Hypercoagulability of COVID-19 patients in intensive care unit. A report of thromboelastography findings and other parameters of hemostasis. J Thromb Haemost 2020. Doi: 10.1111/jth.14850

11 Wright FL, Vogler TO, Moore EE, et al. Fibrinolysis shutdown correlation with thromboembolic events in severe COVID-19 infection. J Am Coll Surg 2020. Doi: 10.1016/j.jamcollsurg.2020.05.007

12 Maatman TK, Jalali F, Feizpour C, et al. Routine venous thromboembolism prophylaxis may be inadequate in the hypercoagulable state of severe coronavirus disease 2019. Crit Care Med 2020. Doi: 10.1097/CCM.0000000000004466

13 Mortus JR, Manek SE, Brubaker LS, et al. Thromboelastographic results and hypercoagulability syndrome in patients with coronavirus disease 2019 who are critically ill. JAMA Netw Open 2020; 3(06):e2011192

14 Pavoni V, Gianesello L, Pazzi M, Stera C, Meconi T, Frigieri FC. Evaluation of coagulation function by rotation thromboelastometry in critically ill patients with severe COVID-19 pneumonia. J Thromb Thrombolysis 2020. Doi: 10.1007/s11239-020-02130-7

15 Gurbel PA, Bliden KP, Guyer K, et al. Platelet reactivity in patients and recurrent events post-stenting: results of the PREPARE POSTSTENTING Study. J Am Coll Cardiol 2005;46(10):1820-1826

16 Kahn SR, Lim W, Dunn AS, et al. Prevention of VTE in nonsurgical patients: Antithrombotic Therapy and Prevention of Thrombosis, 9th ed: American College of Chest Physicians EvidenceBased Clinical Practice Guidelines. Chest 2012;141(2 Suppl): e195S-e226S

17 Barnes GD, Burnett A, Allen A, et al. Thromboembolism and anticoagulant therapy during the COVID-19 pandemic: interim clinical guidance from the anticoagulation forum. J Thromb Thrombolysis 2020;50(01):72-81 